\title{
Desarrollo del pensamiento geográfico: un desafío para la formación docente en Geografía'
}

\author{
Fabián Araya Palacios² y Lana de Souza Cavalcanti
}

\begin{abstract}
RESUMEN
El desarrollo del pensamiento geográfico constituye uno de los temas más relevantes de la educación geográfica actual. Según la literatura científica, esta temática es clave para la formación de ciudadanos geográficamente informados. Sin embargo, faltan estudios empíricos relacionados con el desarrollo del pensamiento geográfico durante la formación de los profesores de geografía. El objetivo del artículo, es presentar los resultados de una investigación realizada como un estudio de caso, cuya finalidad fue indagar sobre algunas categorías que permitan evidenciar los procesos de construcción del pensamiento geográfico. Se trabajó con una muestra de 45 estudiantes de profesorado en Geografía de la Universidad Federal de Goías, Brasil. Metodológicamente, se realizó un análisis de contenido de los trabajos didácticos realizados por los estudiantes durante su práctica profesional. Entre los principales resultados, se aprecia que los trabajos didácticos contienen actividades que, según los planteamientos de Vigotsky, permiten el desarrollo de conceptos y habilidades de pensamiento superior, con énfasis en procesos de razonamiento geográfico.
\end{abstract}

Palabras claves: Educación geográfica, didáctica de la geografía, docencia de la geografía, pensamiento geográfico, razonamiento espacial.

\begin{abstract}
Geographical thinking development is one of the main current topics in geography education. Though, according to the scientific literature, this is key for educating geographically informed citizens, empirical studies on the subject in geography teacher training are limited. This paper reports the results from a case study that examined some categories that may help evidence the development of geographical thinking. For this study, a sample of 45 students from the Geography Teaching Program of the Federal University of Goías, Brazil, was selected. The research method consisted in analyzing the contents of learning activities developed by these students during their teaching practice. Our findings showed that the learning activities contain items that, according to Vigotsky, may favor concept understanding and high-level thinking development, with an emphasis on geographical thinking processes.
\end{abstract}

Keywords: Geography education, geography pedagogy, geography teaching, geographical thinking, spatial reasoning.

Fabián Araya agradece al proyecto de mejoramiento institucional (PMI-FIP-ULS No 1501) y al proyecto IPA Nº 1641. Dirección de Investigación y Desarrollo de la Universidad de La Serena (DIDULS), por el apoyo brindado para la elaboración del presente trabajo. Artículo recibido el 13 de septiembre de 2017, aceptado el 26 de marzo de 2018 y corregido el 6 de junio de 2018.

Departamento de Ciencias Sociales, Universidad de La Serena (Chile). E-mail: faraya@userena.cl

3 Laboratorio de Educación en Geografía, Universidad Federal de Goiás (Brasil). E-mail: Is.cavalcanti@uol.com.br 
La educación geográfica es un área del conocimiento educativo que integra el saber pedagógico y el conocimiento geográfico. Se preocupa del desarrollo del pensamiento geográfico y la formación de una racionalidad geográfica que indague sobre las problemáticas sociales y ambientales que afectan al ser humano en su vinculación con la naturaleza (Rodríguez, 2000; Souto, 2012; Brooks, 2017). La didáctica de la Geografía, por su parte, constituye una subdisciplina de la didáctica general, fundada en el Siglo XVII por Juan Amós Comenio (2007), la cual se nutre del saber pedagógico y del conocimiento geográfico. Inicialmente el foco de la didáctica de la Geografía estaba centrado en las estrategias para mejorar la enseñanza de los conocimientos geográficos, pero con el avance de la psicología educacional y otras disciplinas preocupadas por el desarrollo cognitivo, el foco se ha trasladado hacia el mejoramiento de los procesos de aprendizaje de los estudiantes tanto en la enseñanza formal, no formal e informal.

Actualmente, tanto la educación geográfica como la didáctica de la Geografía, comparten la inquietud por el desarrollo del pensamiento geográfico de los actuales y futuros ciudadanos (Araya, 2013). Los estudios sobre el desarrollo cognitivo han permitido lograr un avance en este propósito y también ha despertado interés por la incorporación de las habilidades cognitivas en el proceso de enseñanza-aprendizaje de la Geografía (Amestoy, 2002; Beas, 2003; Gersmehl, 2008; Lee, 2012; Stuart, 2013; Muñiz, 2017). Por ello, los países desarrollados están incorporando en su currículum mayores y mejores conocimientos sobre Geografía y su enseñanza; se ha comprendido que el conocimiento espacial sobre los territorios y los lugares es un conocimiento estratégico para el ciudadano, pues permite formar personas capaces de comprender los sistemas espaciales y ambientales desde una perspectiva territorial (Gallagher, 2012).

De acuerdo a la literatura especializada, la educación geográfica no ha logrado desarrollar cabalmente en los estudiantes de pedagogía su pensamiento geográfico y las habilidades de pensamiento superior que les permitan comprender integralmente el espacio geográfico y las relaciones sociedad-naturaleza (Araya, 2010; Arenas, 2011; Young, 2015; Jones, 2017). Además, faltan estudios empíricos relacionados con el desarrollo del pensamiento geográfico durante la formación de los profesores de Geografía. El desconocimiento del valor conceptual de la Geografía como disciplina académica para conocer, comprender y representar el mundo actual, acarrea, por ende, la poca valoración de las habilidades geográficas que se utilizan y desarrollan en el campo profesional y académico. Abordar estas cuestiones supone el fortalecimiento de la educación geográfica y de su didáctica, como una disciplina capaz de proporcionar marcos teórico-metodológicos apropiados para la enseñanza de las problemáticas socio-ambientales desde una perspectiva multiescalar (Arenas, 2016).

El artículo, tiene como objetivo presentar los resultados de una investigación realizada, a través de un estudio de caso, cuya finalidad ha sido indagar sobre algunas categorías que permitan evidenciar los procesos de construcción del pensamiento geográfico en estudiantes de profesorado en Geografía de la Universidad Federal de Goías, Brasil ${ }^{4}$. Para ello, se ha realizado un análisis de contenido de los trabajos didácticos realizados por los estudiantes durante su práctica profesional.

El trabajo corresponde a una sección de una investigación postdoctoral relacionada con los procesos y mecanismos de construcción del pensamiento geográfico, desarrollada durante los años 2015 y 2016 en el Instituto de Estudios Socioambientales de la Universidad Federal de Goias (UFG), Brasil, bajo la orientación de la Dra. Lana de Souza Cavalcanti. 
El escrito se organiza en cuatro secciones. En la primera sección, se exponen los conceptos que corresponden al marco teórico del estudio. Se enfatiza la perspectiva histórico-cultural de Vigotsky y el concepto de pensamiento geográfico. En la segunda sección, se desarrolla el marco metodológico en el cual se describe el problema y pregunta de investigación, los objetivos, el tipo de estudio realizado y los procedimientos específicos del proceso investigativo. ${ }^{5}$ En la tercera sección, se describen los resultados y discusión del estudio y finalmente se presentan las conclusiones. A través del trabajo, se espera contribuir con el propósito de integrar el desarrollo del pensamiento geográfico en la formación de los futuros profesores de geografía.

\section{Construcción de conceptos desde la perspectiva histórico-cultural de Vigotsky}

El trabajo docente es una actividad compleja que involucra diferentes dimensiones de conocimiento, entre ellas están el contenido y los fundamentos pedagógico-didácticos para enseñarlo. Con respecto a esos fundamentos, los autores de este artículo conciben el proceso de enseñanza en la perspectiva de la psicología histórico-cultural, por considerar que ella contribuye significativamente para la compresión de ese proceso en su complejidad. En esa perspectiva, se entiende la enseñanza-aprendizaje como un proceso de conocimiento del estudiante, con la mediación didáctica del profesor. En este proceso, la Geografía escolar y los contenidos por ella compartidos son instrumentos simbólicos para la relación del sujeto con el mundo. La Geografía es una de las posibilidades de lecturas de la realidad: la lectura espacial. En este sentido, se afirma que el objetivo de la enseñanza de Geografía es el de contribuir para el desarrollo del pensamiento geográfico del alumno.

En la perspectiva histórico-cultural, una meta importante del trabajo docente es la formación de conceptos, por cuanto se trata de una habilidad fundamental para la vida cotidiana; los conceptos son instrumentos simbólicos importantes para mediar la relación de la gente con la realidad. Vygotsky, destacado representante de esa perspectiva teórica, contribuye en el análisis del aprendizaje y en el desarrollo de las funciones psicológicas superiores. Los conceptos son generalizaciones elaboradas sobre esa realidad. Son herramientas culturales que representan mentalmente un objeto. Son formulados por sucesivos procesos de generalizaciones en el pensamiento, al mismo tiempo que, una vez formados en la mente del sujeto, se tornan conocimientos que generalizan sus experiencias.

Por lo tanto, para la construcción de conceptos la psicología histórico-cultural de Vigotsky ofrece un soporte teórico adecuado a los propósitos del presente trabajo. Este autor propone que el conocimiento escolar se construye por la confrontación entre conceptos cotidianos y conceptos científicos. Según Vigotsky (2000), los conceptos cotidianos están ligados al encuentro inmediato con los objetos empíricos. Los conceptos científicos, al contrario, parten de una abstracción. Al respecto Vigotsky afirma:

Para mayor claridad en la presentación del trabajo, se ha decidido integrar el problema y objetivos de la investigación en la sección de metodología. 
"El desarrollo de los conceptos científicos comienza en el campo de la conciencia y de la arbitrariedad y continua adelante, creciendo de arriba para abajo en el campo de la experiencia personal y de la concreción. El desarrollo de los conceptos científicos comienza en el campo de la concreción y del empirismo y su movimiento es en el sentido de las propiedades superiores de los conceptos: de la conciencia y de la arbitrariedad" (Vigotsky, 2000:350)

En la Geografía, algunos conceptos son fundamentales para la estructura del pensamiento particular de esta ciencia, y, en consecuencia, para orientar el abordaje de los contenidos escolares. Entre ellos, destacan: paisaje, lugar, territorio y naturaleza. Estos conceptos constituyen una buena referencia para la estructuración y selección de los contenidos curriculares. Estos conceptos permiten superar los límites de la enseñanza empirista y mecanicista, aún muy presente en las escuelas, por cuanto, permiten cruzar el umbral de la mera descripción. Para la formación de conceptos, en el caso específico de la Geografía escolar, se trata de articular conceptos geográficos formulados por la ciencia de referencia y aquellos formados, por los alumnos, en el contexto cotidiano, con el objetivo de que ellos desarrollen modos de pensar particulares.

Según Vigotsky, las posibilidades para el desarrollo del pensamiento, propiciadas a través de la escuela, depende, en gran medida, de la calidad de los instrumentos mediadores, destacándose entre ellos el lenguaje. Para este autor, un concepto expresado por una palabra representa una generalización, pero, "cuando una palabra nueva, ligada a un determinado significado, es aprehendido por un niño, su desarrollo está apenas comenzando" (Vigotsky, 2000:246). En este proceso, la memorización de palabras y su asociación con los objetos no lleva, por sí solo, a la formación de conceptos. Las funciones psíquicas superiores (los pensamientos) son mediados por el docente e incorporan en su estructura la utilización de signos. En el proceso de formación de los conceptos, ese signo es la palabra.

La Geografía, como disciplina científica, contribuye a desplegar modalidades de razonamiento espacial que permiten que los seres humanos desarrollen su pensamiento geográfico. Algunas de estas modalidades pueden ser conceptos geográficos o categorías de análisis espacial. Tanto los conceptos como las categorías contribuyen a desarrollar habilidades superiores de pensamiento que, paulatinamente, permiten a las personas adquirir una determinada manera de observar e interpretar la realidad socio-espacial e internalizar una manera específica de conocimiento geográfico. "Se puede reconocer a la Geografía como campo de investigación de la espacialidad de las personas, grupos y estructuras sociales más amplias. Implica entender la relevancia de esas espacialidades para el entendimiento de la dinámica de la vida social, analizada desde una dimensión multiescalar" (Barbosa \& Cavalcanti, 2011:16-17).

Lamentablemente, la investigación sobre el aprendizaje geográfico y sobre el desarrollo de habilidades de pensamiento geográfico (sobre todo en la formación de profesores de Geografía) es aún muy reducido (Bednarz, 2000; Arenas, 2011; Downs, 2011). Esto significa que, si bien existe un relativo consenso respecto de lo que implica aprender Geografía en la actualidad, todavía no

Las citas originales de Vigotsky están en portugués. Fueron traducidas, de manera libre, al español por la Dra. Lana de Souza Cavalcanti con el objetivo de facilitar su comprensión. 
existe acuerdo sobre el contenido (en este caso, tipo de habilidades geográficas) que ese aprendizaje requiere.

\section{Desarrollo del pensamiento geográfico}

La educación geográfica, entre sus propósitos principales, debe propiciar que la relación sociedad-naturaleza se desarrolle sobre la base de una perspectiva integrada del espacio geográfico (Gallagher, 2012; Commission of Geographical Education, 2014). Para lograr este propósito, se requieren mayores conocimientos que promuevan el desarrollo del pensamiento geográfico y habilidades que permitan tomar decisiones razonadas en el contexto espacial local y global.

El pensamiento geográfico corresponde a un conjunto de relaciones sociales y naturales cuyo objetivo es que los estudiantes integren la información adquirida, básicamente a través de los sentidos, en una estructura de conocimiento que tenga pertinencia para ellos (Stuart, 2013). Actualmente, en el contexto cultural anglosajón, el concepto desarrollado para definir y profundizar este proceso se denomina "Geographical Thinking". Ello implica investigar sobre las diversas modalidades y estrategias para desarrollar el pensamiento geográfico, desde la perspectiva social, didáctica y curricular (Gersmehl, 2008; Butt, 2011; Lee, 2012; Stuart, 2013).

El pensamiento geográfico, ha sido definido por National Research Council (2006), como un conjunto de habilidades cognitivas que comprenden formas declarativas, habilidades de percepción del conocimiento espacial y algunas operaciones cognitivas que se pueden utilizar para analizar, comprender, transformar y producir nuevas formas de conocimiento espacial. Asimismo, esta institución señala que el conocimiento espacial integra el aprendizaje y uso combinado de tres elementos claves: los conceptos de espacio, las herramientas de representación y las relaciones entre los elementos, y los procesos de razonamiento. Es innegable el rol que juega la mediación docente para el fortalecimiento de las habilidades espaciales en el aprendizaje de la geografía.

Según Cavalcanti, el pensamiento geográfico contribuye con la contextualización del propio alumno como ciudadano del Mundo. Le permite visualizar, espacialmente, los fenómenos y conocer el Mundo en el que vive, desde la escala local, regional, nacional y mundial. El conocimiento geográfico es indispensable en la formación de individuos participantes de la vida social, en la medida que propicia el entendimiento del espacio geográfico y su rol en las prácticas sociales. “Hay un carácter de espacialidad en toda práctica social, así como un carácter social de la espacialidad" (Cavalcanti, 2014: 11).

El pensamiento geográfico tiene un "poder" relevante para el conocimiento de los lugares y los territorios. Las personas que poseen este tipo de conocimiento tienen la capacidad de analizar integralmente las dimensiones espaciales y logran conceptualizar adecuadamente los diversos impactos que las acciones humanas tienen en el espacio geográfico. Comprender cómo se ad-

Recientemente, la editorial Springer ha publicado el libro "The Power of Geographical Thinking". En el trabajo se analiza el concepto de pensamiento geográfico y se plantean sugerencias para desarrollar este tipo de pensamiento en diferentes países y culturas. Para mayor información revisar en las referencias bibliográficas Brooks (2017). 
quiere este tipo de pensamiento y determinar las claves para su desarrollo resulta interesante por su potencialidad epistemológica y didáctica.

Desde un punto de vista educativo, el desarrollo del pensamiento geográfico corresponde a un tópico importante para la didáctica de los profesores de geografía. La formación actual de profesores y licenciados de geografía debería propiciar la adquisición de habilidades superiores de pensamiento y no solo la memorización de conocimientos que, posiblemente, sean olvidados en un corto o mediano plazo.

La importancia de la Geografía para la formación de los docentes, consiste en la aprehensión de la realidad desde el punto de vista de la espacialidad. Es decir, en la comprensión del papel del espacio en las prácticas sociales y de éstas en la configuración del espacio. La ampliación de los conocimientos más allá del sentido común, la confrontación de los diversos tipos de conocimiento y el desenvolvimiento de capacidades operativas de pensamiento son procesos que pueden ser potenciados con prácticas intencionadas de intervención pedagógica (Cavalcanti, 2014).

La formación y adquisición de conceptos constituye un proceso relevante para el desarrollo del pensamiento geográfico. Ello ha originado una didáctica conceptual que propende a la búsqueda de la pertinencia y profundidad del aprendizaje geográfico.

\section{La formación de conceptos para el desarrollo del pensamiento geográfico}

Actualmente, existen diversas demandas para el trabajo docente en la enseñanza de la geografía. Una de ellas, lo constituye el proceso de formación de un pensamiento geográfico, que permita a los estudiantes comprender el Mundo en el cual se encuentran insertos. Con frecuencia los alumnos no consiguen formar un pensamiento geográfico, necesario para su participación activa en la sociedad. No entienden la importancia de los contenidos geográficos para sus vidas. Por ello, surgen una serie de interrogantes: ¿Por qué es importante el desarrollo del pensamiento geográfico? ¿En qué consiste ese desarrollo? ¿Cómo se procesa en los diferentes niveles y series de enseñanza? ¿Cómo tratar los contenidos de geografía de modo que se permita ese desenvolvimiento?

La respuesta a estas interrogantes podría ayudar a superar el formalismo dominante en la enseñanza, en la medida en que permita al profesor concebir en forma más eficaz su actividad docente y sus competencias de promover ayuda pedagógica a los alumnos en la construcción de su propio razonamiento, para ir más allá de la mera transmisión de los contenidos estipulados en los libros didácticos y programas de estudio.

El proceso de enseñanza, busca el desarrollo de determinadas capacidades cognitivas por medio de la formación de conceptos sobre la temática estudiada. Por lo tanto, se requiere el dominio de conceptos específicos de esa disciplina y de su lenguaje propio. Según Cavalcanti, la Geografía desenvuelve un lenguaje, un cuerpo conceptual que acaba por constituirse en un lenguaje geográfico. Ese lenguaje está permeado por conceptos que son requisitos para el análisis de los fenómenos desde el punto de vista geográfico (Cavalcanti, 2014). 
“Enseñar a pensar, ayudar a los alumnos a potenciar sus habilidades cognitivas, proveer a los alumnos de instrumentos conceptuales para la construcción de conocimientos son tareas que requieren del profesor una comprensión de esos procesos de modo que se realice su trabajo con mayor eficacia" (Cavalcanti, 2014:174).

A través de la formación de conceptos, tanto el conocimiento científico como el conocimiento escolar contribuye a la formación del pensamiento geográfico. En este sentido, siguiendo a Shulman (1993), el conocimiento didáctico del contenido, como concepto base, propicia la integración de ambos tipos de conocimiento y contribuye también con el desarrollo del pensamiento geográfico. Los conceptos seleccionados componen un sistema conceptual más amplio en la estructuración de un pensamiento geográfico que deben ser considerados en sus interrelaciones.

\section{Procedimientos didácticos para la construcción de conceptos}

La didáctica de la Geografía, al igual que otras didácticas específicas, tiene su origen en la preocupación sobre "cómo enseñar" los contenidos específicos. Aunque la didáctica general, cronológicamente anterior, pretendía dar respuesta a los problemas de cómo enseñar de manera general, a lo largo del tiempo se fue mostrando la necesidad de una reflexión específica sobre la enseñanza y el aprendizaje de cada área disciplinaria. Esta necesidad fue formulada por Lee Shulman (1993) quien, a finales de la década de 1970, denominó el paradigma ausente a la inexistencia de un campo de investigación sobre los problemas de aprendizaje de los contenidos (Souto, 2013).

Según Bolívar (2005), Shulman ha sido un autor relevante para el desarrollo de las didácticas específicas, por cuanto considera que la enseñanza (en este caso de la Geografía) enfatiza la comprensión y el razonamiento, la transformación y la reflexión. Un aspecto esencial del concepto de enseñanza para este autor, lo constituye el propósito de que los alumnos logren comprender y resolver problemas, que aprendan a pensar crítica y creativamente y que comprendan datos, principios y normas de procedimiento (Shulman, 2005). Uno de los conceptos centrales de la propuesta de Shulman lo constituye el conocimiento didáctico del contenido, esa especial relación entre contenido específico y pedagogía que constituye una esfera exclusiva del conocimiento de los profesores, es decir, su propia forma de comprensión profesional.

En el caso de la enseñanza de la Geografía, uno de los procesos específicos que los docentes deben internalizar durante su formación inicial (para posteriormente trabajarlo con sus estudiantes), corresponde al desarrollo del pensamiento geográfico. Para ello, resulta relevante la vinculación entre los conceptos geográficos (conocimiento del contenido) y la forma de enseñar esos conceptos (conocimiento didáctico del contenido), con el propósito de promover entre los estudiantes la capacidad de razonar y pensar geográficamente. Para lograr este objetivo, es necesario conocer y utilizar estrategias didácticas que propicien este proceso de manera intencionada y sistemática. 


\section{Estrategias didácticas para el desarrollo del pensamiento geográfico}

Según Campos (2011), Vigotsky plantea procedimientos didácticos para la construcción de conceptos. Entre ellos destacan:

1. Problematización de las prácticas y los saberes espaciales de los alumnos (un cuestionamiento, una tarea a ser resuelta).

2. Método de dupla estimulación en el cual dos conjuntos de estímulos son presentados al sujeto, uno como objeto de su actividad y otro como signos (palabras, mapas, textos etc.)

3. Dirigir la acción pedagógica sobre la zona de desenvolvimiento proximal.

4. Crear desafíos que serán difíciles y al mismo tiempo posibles de ser realizados por los alumnos y que tales dificultades solo sean superadas a través de aprendizajes de nuevos contenidos.

5. Desarrollar un sistema jerárquico de inter-relaciones que caracterizan los conceptos científicos, proceso mediante el cual el concepto de espacio, por ejemplo, se pueda convertir en territorio, lugar, paisaje, etc.

Vigotsky inspira tales procedimientos en sus investigaciones, específicamente, sobre el proceso de formación de conceptos, en que trabaja la forma en la cual el raciocinio y el contenido del pensamiento de los niños son transformados. Desde el punto de vista de los procesos psicológicos, el concepto es una generalización, en la medida que encarna la articulación de los momentos de universalidad con el de singularidad, pasando por la particularidad. Para Vigotsky, la construcción de conocimientos es un proceso esencialmente social e histórico, y tiene su explicación en la compleja relación entre pensamiento y lenguaje, el que ha de ser comprendido en sus raíces filogenéticas y ontogenéticas (Vigotsky, 2000).

Cuando una persona desea aprender un nuevo concepto, ello presupone el desarrollo de muchas funciones intelectuales: atención deliberada, memoria lógica, abstracción, capacidad para comparar y diferenciar. Por ello, según Vigotsky, la enseñanza directa de conceptos es imposible. Lo que hay que hacer en la educación es crear oportunidades para que los nuevos conceptos sean desarrollados. La idea es que primero los conceptos sean utilizados en la construcción de determinados raciocinios y en la problematización de las prácticas sociales de los alumnos, para después ser definidos.

Para el caso de la educación geográfica, la formación de conceptos se relaciona con estrategias didácticas que permitan la construcción de conocimientos geográficos en un contexto real, en una práctica situada, al modo como lo hacen los especialistas en Geografía o geógrafos. En palabras de Gersmelh \& Gersmelh (2007), el pensamiento geográfico corresponde al conjunto de habilidades que usan los geógrafos para analizar las relaciones espaciales.

Una estrategia didáctica adecuada para desarrollar el pensamiento geográfico, consiste en profundizar la habilidad de describir y explicar el funcionamiento de un territorio, considerando las dimensiones espaciales de un evento o problema que afecta a la sociedad. Desarrollar esta habilidad (y otras habilidades asociadas al razonamiento geográfico) permitiría, según Stuart (2013),

Ver especialmente el capítulo $N^{\circ}$ 4: Las raíces genéticas del pensamiento y del lenguaje, p. 111-150. 
contar con un repertorio de conceptos espaciales, herramientas de representación y procesos de razonamiento espacial, los cuales, movilizados conjuntamente, permitirían no solo la comprensión del espacio, sino que, a su vez, la resolución de problemas espaciales y el desarrollo de la conciencia espacial.

En este trabajo, se categorizarán los cinco procedimientos propuestos por Vigotsky y citados por Campos (2011), con la finalidad de verificar su presencia o ausencia en las estrategias didácticas propuestas por los estudiantes de pedagogía en Geografía en sus trabajos de práctica obligatoria. Se entenderá que aprender Geografía, de manera activa, significa adquirir y desarrollar habilidades relacionadas con el pensamiento geográfico, las cuales deben permitir a las personas resolver problemas espaciales en contexto geográfico, comprendiendo el funcionamiento de un territorio y adquiriendo conciencia de su responsabilidad en el espacio geográfico.

\section{Aspectos metodológicos de la Investigación}

La investigación, desde el punto de vista de los fines extrínsecos o externos, correspondió a una investigación de tipo aplicada. Pretendió contribuir con la descripción de las experiencias y estrategias didácticas para el desarrollo del pensamiento geográfico y habilidades cognitivas utilizadas por los futuros profesores de geografía en sus trabajos de práctica. Por otro lado, desde el punto de vista de los fines intrínsecos o internos, la presente investigación correspondió a un estudio de caso de carácter descriptivo, pues, reunió y sistematizó información relacionada con los trabajos desarrollados por un grupo acotado de estudiantes en sus prácticas obligatorias.

\section{Procedimientos de la investigación}

En primer lugar, se planteó el siguiente problema de investigación: ¿Cuáles son las categorías que evidencian los procesos de construcción del pensamiento geográfico en los trabajos didácticos de los estudiantes de práctica obligatoria de la Licenciatura en Geografía de la Universidad Federal de Goias (UFG)? ${ }^{9}$

Se planteó una investigación de carácter cualitativa. Se definió, para ello, una pregunta de investigación que orientara, de manera más específica, el proceso de búsqueda y análisis de la información. La pregunta se planteó de la siguiente manera: ¿cuáles son las estrategias didácticas relacionadas con el pensamiento geográfico, utilizadas por los estudiantes de práctica obligatoria de la licenciatura en geografía en sus trabajos didácticos de clases?

Con la finalidad de operacionalizar esta pregunta, se definieron los siguientes objetivos generales y específicos de investigación:

Objetivo general: comprender las categorías que evidencian los procesos de construcción del pensamiento geográfico en los trabajos didácticos de los estudiantes de práctica obligatoria de la Licenciatura en Geografía de la UFG. 


\section{Objetivos específicos}

1. Relacionar las estrategias didácticas utilizadas por los estudiantes de Licenciatura en sus prácticas pedagógicas, con los procesos de formación del pensamiento geográfico.

2. Interpretar la información conceptual y empírica recolectada, en relación con los procesos de formación del pensamiento geográfico.

En segundo lugar, se definió el diseño de la investigación. La investigación utilizó un diseño de investigación no experimental. Una de las características del diseño no experimental es que los sujetos no son asignados al azar a los grupos, ni emparejados; sino que dichos grupos ya estaban formados antes del experimento, son grupos intactos (Hernández, 2010). Este diseño se utilizó para organizar el proceso de investigación de acuerdo a cada una de sus fases y etapas. Permitió sistematizar la indagación y reunir e interpretar la información cualitativa surgida a medida que se desarrollaba el proceso de investigación.

En tercer lugar, se especificó la muestra. Considerando que la muestra contiene las características de la población y de acuerdo con los objetivos de la investigación, se seleccionó una muestra de tipo intencional de carácter no probabilística. La muestra se conformó por un grupo de 45 estudiantes de Licenciatura en Geografía de la Universidad Federal de Goias. Correspondió al curso denominado "Estagio Supervisonado em Geografia II" y se compuso de 29 hombres y 16 mujeres. El curso se encontraba en el tercer año de su carrera y, por lo tanto, los estudiantes habían tenido experiencia teórico-metodológica en los temas de su especialidad y también experiencia práctica en las observaciones de clases desarrolladas en la enseñanza fundamental (primaria) y en la enseñanza media (secundaria).

En cuarto lugar, se definieron los instrumentos de recolección de información y se aplicaron a la muestra seleccionada. Es necesario señalar que, dado que el proyecto inicial era de mayor envergadura y extensión fueron aplicados tres instrumentos de recolección de información (cuestionario semiestructurado, focus group y análisis de trabajos didácticos). En el presente trabajo, con la finalidad de lograr una mayor focalización temática, solo se ha considerado el análisis de las categorías de los trabajos didácticos elaborado por los estudiantes de pedagogía durante sus prácticas obligatorias.

Para la elaboración del instrumento de análisis de las categorías, se consideraron los procedimientos didácticos para la construcción de conceptos propuestos por Vigotsky (citado por Campos, 2011), en el cual se plantean cinco procedimientos específicos. De acuerdo con lo anterior, las categorías definidas fueron las siguientes: problematización de las prácticas y los saberes espaciales de los alumnos; Método de dupla estimulación; Acción pedagógica sobre la zona de desenvolvimiento proximal; Desafíos difíciles y posibles de ser realizados por los estudiantes y desarrollo de un sistema jerárquico de inter-relaciones que caracterizan los conceptos científicos.

Finalmente, a través de una matriz digital, se analizaron los trabajos elaborados por los estudiantes durante su práctica obligatoria. Esta información se sometió a un análisis de contenido, que permitió la estructuración de textos homogéneos que dieron cuenta de la identificación de las categorías en las propuestas didácticas de los estudiantes. Con la finalidad de guiar el análisis 
de los textos, se consideró la pregunta de investigación, especialmente en lo concerniente con las estrategias didácticas relacionadas con el pensamiento geográfico. En la siguiente sección, se presentan, a modo de ejemplo, algunas de las respuestas de los estudiantes y su relación con las categorías seleccionadas.

\section{Análisis de los trabajos según los procedimientos didácticos para la construcción de conceptos propuestos por Vigotsky}

En el Cuadro $N^{\circ} 1$, se presenta la información emanada de los trabajos didácticos realizados por los estudiantes de Geografía, relacionados con los procedimientos pedagógicos que Vigotsky considera relevantes para la construcción de conceptos (Campos, 2011).10

Cuadro $\mathrm{N}^{\circ} 1$

Procedimientos didácticos para la construcción de conceptos, según Vigotsky.

\begin{tabular}{|l|l|}
\hline $\begin{array}{l}\text { Procedimientos } \\
\text { didácticos según } \\
\text { Vigotsky }\end{array}$ & \multicolumn{1}{c|}{$\begin{array}{c}\text { Evidencias de los trabajos didácticos de los estudiantes de } \\
\text { Licenciatura (pedagogía) en Geografía. }\end{array}$} \\
\hline $\begin{array}{l}\text { Problematización de } \\
\text { las prácticas y los } \\
\text { saberes espaciales } \\
\text { de los alumnos (Ej. un } \\
\text { cuestionamiento, una } \\
\text { tarea a ser resuelta). }\end{array}$ & $\begin{array}{l}\text { Alumno 1. "Metodología de enseñanza: movilización de contenidos; } \\
\text { Luego la construcción del sujeto de su subjetividad a través de la } \\
\text { dialéctica, de esa forma el pensamiento teórico-conceptual es carac- } \\
\text { terístico de la subjetividad humana poseedor de una naturaleza social } \\
\text { e histórica. Es un proceso complejo que ocurre con la internalización } \\
\text { de algunas experiencias sociales y culturales". }\end{array}$ \\
$\begin{array}{l}\text { Alumno 2. "Como la cartografía ha sido trabajada en la sala de clases } \\
\text { con el objetivo de que los alumnos tengan claridad de la capacidad de } \\
\text { representación de los fenómenos, análisis, interpretación y lectura". }\end{array}$ \\
$\begin{array}{l}\text { Alumno 3. "Uso de la secuencia didáctica como una metodología ins- } \\
\text { trumentalizadora de las acciones. Primera aula': problematización so- } \\
\text { bre continente antártico con el objetivo de comprender la percepción } \\
\text { y los conocimientos previos de los alumnos de la temática, analizando } \\
\text { las imágenes de los libros didácticos relacionadas con la antártica. } \\
\text { Utilizando un mapa-mundi se demuestra la localización del continente } \\
\text { antártico y la percepción espacial de los alumnos; Segunda aula: exi- } \\
\text { bición de video y aplicación de una actividad discursiva, solicitando } \\
\text { que los alumnos expongan la importancia de la antártica en las cues- } \\
\text { tiones ambientales y políticas del mundo". }\end{array}$ \\
\hline
\end{tabular}

Las evidencias de los trabajos didácticos de los estudiantes de Licenciatura en Geografía, corresponden solamente a ejemplos que permitan visualizar las actividades propuestas y su relación con los procedimientos didácticos planteados por Vigotsky. 
Continuación Cuadro $N^{\circ} 1$

\begin{tabular}{|c|c|}
\hline $\begin{array}{l}\text { ntos } \\
\text { según }\end{array}$ & $\begin{array}{l}\text { Evidencias de los trabajos didácticos de los estudiantes de } \\
\text { Licenciatura (pedagogía) en Geografía. }\end{array}$ \\
\hline & $\begin{array}{l}\text { Alumno 4. "Metodología: aula expositiva y dialogada compuesta por } \\
\text { tres momentos: - problematización con ayuda de imágenes y pregun- } \\
\text { tas. - para relacionar contenido con geografía: utilización de concep- } \\
\text { tos de estado, territorio y región. -Explicación con visualización en } \\
\text { mapas de los motivos de la disputa por el territorio a través del grupo } \\
\text { denominado Estado Islámico". } \\
\text { Alumno 5. "Al final del aula los alumnos ya poseían otras visiones de } \\
\text { la temática abordada de toda una dinámica política y la visión que no } \\
\text { es la religión la causa principal de esa disputa, sino una disputa por } \\
\text { territorios y por la conquista de más poder". }\end{array}$ \\
\hline $\begin{array}{l}\text { Método de dupla } \\
\text { estimulación en el } \\
\text { cual dos conjuntos de } \\
\text { estímulos son presen- } \\
\text { tados al sujeto, uno } \\
\text { como objeto de su } \\
\text { actividad y otro como } \\
\text { signos (Ej. palabras, } \\
\text { mapas, libros etc.) }\end{array}$ & $\begin{array}{l}\text { Alumno 1. "Percibí que el profesor utiliza mucho el libro didáctico, } \\
\text { pero hace uso de él de forma orientadora e inteligente. El trae textos } \\
\text { complementarios, hechos cotidianos y el uso del mapamundi con el } \\
\text { objetivo de profundizar los contenidos". } \\
\text { Alumno 2. "La enseñanza desde hace mucho dejó de ser pensada } \\
\text { como trasmisión de conceptos, más propone que el alumno se torne } \\
\text { sujeto de su propio proceso de enseñanza-aprendizaje teniendo como } \\
\text { mediador al profesor". } \\
\text { Alumno 3. "Se considera más que todo una práctica del alumno y sus } \\
\text { conocimientos previos y posteriormente relacionados con el conoci- } \\
\text { miento científico a través de salidas de campo". }\end{array}$ \\
\hline $\begin{array}{l}\text { Dirigir la acción peda- } \\
\text { gógica sobre la zona } \\
\text { de desenvolvimiento } \\
\text { proximal }\end{array}$ & $\begin{array}{l}\text { Alumno 1. "Desarrollar en los alumnos la capacidad de observación y } \\
\text { reflexión de su cotidiano". } \\
\text { Alumno } 2 \text {. "Identificando los signos, imágenes, funciones y relaciones } \\
\text { presentes en su cotidiano". } \\
\text { Alumno 3. "Realizamos metodología de tempestad cerebral para le- } \\
\text { vantamiento previo de sus conocimientos". } \\
\text { Alumno 4. "Se procuró establecer la formación y mediación de con- } \\
\text { ceptos que permitieran un análisis y comprensión del continente en la } \\
\text { perspectiva de la geopolítica y física, utilizando algunos conceptos de } \\
\text { geografía: espacio, territorio y región". }\end{array}$ \\
\hline
\end{tabular}


Continuación Cuadro $N^{\circ} 1$

\begin{tabular}{|c|c|}
\hline & $\begin{array}{l}\text { Evidencias de los trabajos didácticos de los estudiantes de } \\
\text { Licenciatura (pedagogía) en Geografía. }\end{array}$ \\
\hline & $\begin{array}{l}\text { Alumno 5. "Redes de interacciones de alumno con su cotidiano, con } \\
\text { foco en entender el medio en que se vive". } \\
\text { Alumno 6. "pude percibir un 'ambiente geográfico" rico en recursos } \\
\text { que elevan la calidad del proceso de enseñanza y aprendizaje para la } \\
\text { disciplina para mejorar la comprensión de los fenómenos geográficos". }\end{array}$ \\
\hline $\begin{array}{l}\text { Crear desafíos que se- } \\
\text { rán difíciles y al mismo } \\
\text { tiempo posibles de } \\
\text { ser realizados por los } \\
\text { alumnos y que tales } \\
\text { dificultades solo sean } \\
\text { superadas a través de } \\
\text { aprendizajes de nue- } \\
\text { vos contenidos. }\end{array}$ & $\begin{array}{l}\text { Alumno 1. "La ciudad resulta de un proceso continuo de construcción } \\
\text { y deconstrucción material por la sociedad". } \\
\text { Alumno 2. "Contribución geográfica para comprender la diversidad } \\
\text { cultural de oriente, con foco en la espacialización que las mujeres re- } \\
\text { presentan en la sociedad musulmana". } \\
\text { Alumno 3. "Fueron escogidos dos conceptos: lugar y territorialidad". }\end{array}$ \\
\hline $\begin{array}{l}\text { Desarrollar un sistema } \\
\text { jerárquico de inter-re- } \\
\text { laciones que carac- } \\
\text { terizan los conceptos } \\
\text { científicos, proceso } \\
\text { mediante el cual el } \\
\text { concepto de espacio, } \\
\text { por ejemplo, se pueda } \\
\text { convertir en territorio, } \\
\text { lugar, paisaje, etc }\end{array}$ & $\begin{array}{l}\text { Alumno1. "En este sentido, los monumentos escultóricos permitie- } \\
\text { ron acciones mediadoras de los principales conceptos geográficos: } \\
\text { espacio, paisaje, ciudad y urbano; como también la interposición de } \\
\text { conceptos más específicos: identidad, memoria, monumento, poder } \\
\text { y representación". } \\
\text { Alumno 2. "Los acontecimientos de la escala local, son ejemplos de } \\
\text { escalas globales y complejas". } \\
\text { Alumno 3. "Capacidad de relacionarlo con un contexto global". } \\
\text { Alumno 4. "Reconocimiento de los elementos que componen la es- } \\
\text { tructura urbana de Goiânia, en el contexto de una escala global-local". } \\
\text { Alumno 5. "Comprensión e importancia de los aspectos socioespacia- } \\
\text { les presentes en el nordeste brasileiro". } \\
\text { Alumno 6. "Al final del aula los alumnos ya poseían otras visiones de } \\
\text { la temática abordada de toda una dinámica política y la visión que no } \\
\text { es la religión la causa principal de esa disputa, sino una disputa por } \\
\text { territorios y por la conquista de más poder". }\end{array}$ \\
\hline
\end{tabular}

Fuente: Elaboración propia. 
Según el Cuadro № 1, la revisión de los trabajos didácticos dejó en evidencia la utilización, por parte de los futuros docentes, de actividades que, según la propuesta de Vigotsky (Campos, 2011), permiten el desarrollo de conceptos y habilidades de pensamiento, con énfasis en procesos de razonamiento geográfico.

- Problematización de las prácticas y los saberes espaciales de los alumnos: se identificaron actividades que promueven la problematización de las prácticas y los saberes espaciales de los estudiantes. Por ejemplo, la utilización de cartografía con el objetivo de que los alumnos tengan claridad de la capacidad de representación de los fenómenos, análisis, interpretación y lectura visual del espacio geográfico. Problematización sobre el continente antártico, con el objetivo de comprender la percepción y los conocimientos previos de los alumnos sobre la temática. Problematización, con ayuda de imágenes y preguntas, para relacionar la geografía con los conceptos de estado, territorio y región. Estas actividades promueven prácticas en las cuales los estudiantes trabajan activamente en "proyectos" y permiten el trabajo colaborativo.

- Método de dupla estimulación: se identificaron actividades que promueven el trabajo de los estudiantes a través del método de dupla estimulación. Por ejemplo, la utilización del libro didáctico, textos complementarios y mapamundi con el objetivo de profundizar los contenidos geográficos. Las preguntas que emergen en una salida de campo y la mediación de los docentes son actividades que logran que los estudiantes construyan relaciones y analicen críticamente los fenómenos producto de la comprensión, búsqueda e interpretación de la información.

- Acción pedagógica sobre la zona de desenvolvimiento proximal: se identificaron actividades que promueven la acción pedagógica sobre la zona de desenvolvimiento proximal. Por ejemplo, desarrollar, en los estudiantes, la capacidad de observación y reflexión del espacio geográfico cotidiano, identificando signos, imágenes, funciones y relaciones espaciales. Utilización de una metodología de tempestad cerebral para la construcción de conceptos geográficos. Percepción de un 'ambiente geográfico" rico en recursos didácticos que permiten elevar la calidad del proceso de enseñanza-aprendizaje de la Geografía. Estas actividades son coincidentes con el aprendizaje de carácter social y con la acción pedagógica sobre la zona de desenvolvimiento proximal propuesta por Vigotsky, como base de su teoría histórico-cultural.

- Desafíos difíciles y posibles de ser realizados por los estudiantes: se identificaron actividades que proponen desafíos difíciles y posibles de ser realizados por los estudiantes. Por ejemplo, trabajar el concepto de ciudad como resultado de un proceso continuo de construcción y deconstrucción material realizado por la acción humana. Conceptualización geográfica para comprender la diversidad cultural de oriente medio, con foco en la construcción del espacio por medio de la acción de las mujeres en la sociedad musulmana. Las evidencias más específicas vinculadas con estrategias tendientes a desarrollar habilidades de razonamiento y actuación en el contexto del pensamiento geográfico, están enfocadas hacia actividades de resolución de problemas, basadas en el planteamiento de situaciones abiertas, que exigen de los alumnos una actitud activa y un esfuerzo por buscar sus propias respuestas. Ello es coincidente con la propuesta de Vigotsky, en el sentido de crear desa- 
fíos que serán difíciles y al mismo tiempo posibles de ser realizados por los estudiantes y que tales dificultades solo sean superadas a través de aprendizajes de nuevos contenidos.

- Desarrollo de un sistema jerárquico de inter-relaciones que caracterizan los conceptos científicos: se identificaron actividades que plantean estrategias didácticas relacionadas con un sistema jerárquico de inter-relaciones que caracterizan los conceptos científicos. Por ejemplo, trabajar con monumentos escultóricos con la finalidad de vincularlos con diversos conceptos geográficos, tales como: espacio, paisaje, ciudad y urbano; como también la interposición de conceptos más específicos: identidad, memoria, monumento, poder y representación. Reconocimiento de los elementos que componen la estructura urbana de la ciudad de Goiânia, en el contexto de una escala global-local. Comprensión e importancia de los aspectos socioespaciales presentes en el nordeste brasileiro. Se constató la relevancia de la construcción de conceptos geográficos. Ello fomenta en los estudiantes la capacidad de aprender a aprender (habilidades meta-cognitivas).

\section{Estrategias didácticas y construcción de conceptos relacionados con el pensamiento geográfico}

En relación con las estrategias didácticas y construcción de conceptos relacionados con el pensamiento geográfico, se desprende lo siguiente:

- Estrategias didácticas: en las propuestas de los estudiantes, se aprecia que las estrategias didácticas son concebidas como un trabajo socio-constructivo que permite la actividad y la construcción de conocimiento por parte del alumno. Sin embargo, no se visualiza un proceso intencionado, a largo plazo, para el desarrollo de modalidades de razonamiento espacial. Según vigotsky, estas habilidades se adquieren a través de la siguiente secuencia: problematización, método de dupla estimulación, acción pedagógica sobre la zona de desarrollo próximo, crear desafios y sistema jerárquico de inter-relaciones. Este es un proceso social e histórico de larga duración que trasciende los objetivos del presente trabajo. Para lograr este objetivo, según Cavalcanti, las estrategias didácticas deben orientarse a contextualizar espacialmente los fenómenos, conocer el Mundo en que se vive multiescalarmente y participar en la vida social (Cavalcanti, 2014).

- Conceptos Geográficos: en las respuestas de los estudiantes, se aprecia que los conceptos geográficos son relevantes para el conocimiento geográfico. Los estudiantes al identificar los elementos que componen el espacio geográfico y al comprender cómo los factores climáticos se relacionan con el tipo de hábitats (por ejemplo, la ciudad de Goiânia y su pertenencia a una región determinada), hacen de la salida a terreno una instancia de aprendizaje propicia para el desarrollo de la comprensión geográfica y la construcción de conceptos, siempre y cuando esté mediada y dirigida a trascender la descripción del paisaje. En palabras de Rodríguez, "Al describir se pinta, se dibuja a través de la palabra; es entonces una representación que involucra objetos, hechos, procesos reales, experiencias, vivencias, lo que se percibe, se recuerda, se siente, se piensa, se imagina, del macro/micro entorno donde el sujeto se desenvuelve. Es vital enfatizar, en el marco de la descripción la impor- 
tancia de establecer las relaciones para comprender los procesos socio espaciales que protagoniza el hombre" (Rodríguez, 2007: 146).

La habilidad de observación es utilizada, por los futuros docentes, en varias de las propuestas didácticas. Según Rodríguez, "La observación es de vital importancia para el desarrollo del pensamiento geográfico, porque implica una tarea de exploración con un propósito específico: descubrir características, rasgos, relaciones, posiciones que posibilitan resolver problemas de conocimiento. No es una tarea simple, ni pasiva, requiere un constante intercambio entre la mente del observador y el objeto observado: el observador interroga y el objeto responde" (Rodríguez, 2007: 143). En los trabajos analizados, la preferencia por las habilidades de observación y en segundo término de comprensión, parece guiar, a su vez, la apertura de los futuros docentes para desarrollar actividades fuera de la sala de clases.

\section{Conclusiones}

Considerando la pregunta de investigación, ¿cuáles son las estrategias didácticas relacionadas con el pensamiento geográfico, utilizadas por los estudiantes de práctica obligatoria de la licenciatura en geografía en sus trabajos didácticos de clases?, se puede señalar, de manera preliminar, que los futuros docentes utilizan diferentes estrategias didácticas para la construcción de conceptos y para el desarrollo del pensamiento geográfico. Las estrategias están orientadas por concepciones sociales y mediadas por las interacciones entre estudiantes y docentes, lo cual es coincidente con las categorías señaladas por Vigotsky. Sin embargo, no se visualiza un proceso intencionado, a largo plazo, para el desarrollo de modalidades de razonamiento espacial, que permitan desarrollar habilidades superiores de pensamiento geográfico. Este es un proceso social e histórico de larga duración que trasciende los objetivos del presente trabajo.

De acuerdo al primer objetivo específico, "relacionar las estrategias didácticas utilizadas por los estudiantes de Licenciatura en sus prácticas pedagógicas, con los procesos de formación del pensamiento geográfico", se puede señalar que las estrategias didácticas identificadas, responden, mayoritariamente, a una concepción socio-constructivista del aprendizaje geográfico. Se orientan, fundamentalmente, hacia las habilidades de observación y comprensión de conceptos espaciales, estrategias de interrelación, representación y razonamiento espacial. Ello implica un gran desafío cognitivo para lograr profundizar en mayores niveles de integración y actuación en el espacio geográfico. En los trabajos analizados, la preferencia por las habilidades de observación y en segundo término de comprensión, parece guiar, a su vez, la apertura de los futuros docentes para desarrollar actividades fuera de la sala de clases. Resulta necesario continuar investigando en esta problemática, con la finalidad de elaborar propuestas fundamentadas empíricamente, que contribuyan con esta importante temática de la educación geográfica actual.

De acuerdo al segundo objetivo específico, "interpretar la información conceptual y empírica recolectada en relación con los procesos de formación del pensamiento geográfico", se puede señalar que el concepto de pensamiento geográfico es complejo y multidimensional. Por un lado, se relaciona con los aspectos cognitivos del ser humano $y$, por otro, con los aspectos sociales y culturales que permiten el desarrollo de las habilidades superiores de pensamiento. Se aprecia la importancia de la formación de conceptos para el desarrollo del pensamiento geográfico, a través 
de la utilización de diversas estrategias didácticas. Existe, para ello, una preocupación y valoración por el desarrollo del pensamiento geográfico. Sin embargo, no hay evidencias específicas que permitan sostener que existe una metodología definida con la cual se pueda desarrollar este pensamiento de una manera precisa y consistente.

Los resultados de la investigación, proporcionan un estímulo para seguir indagando sobre las habilidades de desarrollo del pensamiento geográfico, en el contexto de la educación geográfica actual. Solamente a través de ciudadanos geográficamente informados y de profesores adecuadamente formados, podremos mantener la esperanza de tomar decisiones y actuar correctamente en un mundo cada vez más globalizado e interdependiente.

\section{Referencias bibliográficas}

AMESTOY, M. Research on the development and teaching of thinking skills. REDIE Revista Electrónica de Investigación Educativa, 2002. Disponible en internet:

http:/redie.ens.uabc.mx/vol4no1/contents-amestoy.html

ARAYA, F. Educación geográfica para la sustentabilidad. La Serena: Editorial Universidad de La Serena, 2010.

ARAYA, F. (editor). Formación Ciudadana desde la Educación Geográfica. La Serena: Editorial Universidad de La Serena, Dirección de Investigación, Serie Monografías, 2013.

ARENAS, A. y SALINAS, V. Giros en la Educación Geográfica: renovación de lo geográfico y lo educativo. Revista de Geografía Norte Grande, № 56, 2011, p.143-162.

ARENAS, A.; FERNÁNDEZ, H. y PÉREZ, P. (editores). Una Educación Geográfica para Chile. Santiago de Chile: Sociedad Chilena de Ciencias Geográficas, 2016.

BARBOSA, E. \& CAVALCANTI, L. A cidade, os sujeitos e suas práticas espaciais cotidianas. In: BARBOSA, E. \& CAVALCANTI, L. (editoras). A cidade e seussujeitos. Goiânia: Editorial Vieira, 2011, p.16-29.

BEAS, J.; SANTA CRUZ, J.; THOMSEN, P. y UTRERAS, S. Enseñar a pensar para aprender mejor. Santiago de Chile: Ediciones Universidad Católica de Chile, Tercera edición, 2003.

BEDNARZ, S. Geography Education Research in the Journal of Geography 1988- 1997. International Research in Geographical and Environmental Education, 2000, № 9, p. 88-97.

BOLIVAR, A. Conocimiento didáctico del contenido y didácticas específicas. Profesorado. Revista de currículum y formación del profesorado, 2005, Vol. 2, № 9. Disponible en internet: http://www. ugr.es/ recfpro/rev92ART6.pdf

BUTT, G. (edited). Geography, Education and the Future. Great Britain: continuum International Publishing group, 2011. 
BROOKS, C. International differences in thinking geographically, and why "the local" matters. In: BROOKS, C.; BULL, G. \& FARGHER, M. (editors). The power of geographical thinking. Internacional Perspectives on Geographical Education. USA: Springer International Publishing AG, 2017, p. 169179.

CAMPOS, M. Método dialéticona didáctica da Geografía. En: CAVALCANTI, L.; APARECIDA, M. \& DE SOUZA, V. Produção do conhecimento e pesquisa no ensino da geografia. Goiânia: Editora da PUC, 2011, p.10-19.

CAVALCANTI, L. Geografía, escola e construção de conhecimentos. Campinas: Papirus Editora, $18^{\circ}$ edición. Segunda reimpresión, 2014.

COMMISSION OF GEOGRAPHICAL EDUCATION. Lucerne Declaration. 2014. Disponible en internet: http://www.igu-cge.org/

COMENIO, J. Didáctica Magna. Ciudad de México: Ed. Porrúa, 17a Edición, 2007.

DOWNS, R. El Informe NAEP Geografía 2010: ¿Qué haremos después? Journal of Geography, 2011, Vol. 111, No 1, p. 39-40.

GALLAGHER, S. \& DOWNS, R. (editors). Geography for Life: National Geography Standards. Geography Education National Implementation Proyect (GENIP). Washington: National Council for Geographic Education, 2012.

GERSMEHL, P. Teaching Geography. New York: The Guilford Press, Second Edition, 2008.

GERSMEHL, P. \& GERSMEHL, C. Spatial thinking by young children: neurologic evidence for early development and educability. Journal of Geography, 2007, N 106, p.181-191.

HERNÁNDEZ, R.; FERNÁNDEZ, C. y BAPTISTA, M. Metodología de la investigación. Ciudad de México: Mcgraw-Hill, Quinta edición, 2010.

JONES, M. (edited). The Handbook of Secondary Geography. Bristol: Geographical Association, 2017.

LEE, J. \& BEDNARZ, R. Components of Spatial Thinking: Evidence from a Spatial Thinking Ability Test. Journal of Geography, 2012, Vol. 111, N 1, p. 15-26.

MUÑIZ, O.; SOLEM, M. \& BOEHM, R. Building a Foundation for International Collaborative and Comparative Research on Geography Learning Progressions. In: MUÑIZ, O.; SOLEM, M. \& BOEHM, R. (editors). Learning Progressions in Geography Education: International Perspectives. Internacional Perspectives on Geographical Education. USA: Springer International Publishing AG, 2017, p. V-XIII.

NATIONAL RESEARCH COUNCIL. Learning to Think Spatially. Washington: National Academies Press, 2006. 
RODRÍGUEZ, A. Geografía conceptual: enseñanza y aprendizaje de la geografía en educación básica primaria. Santafé de Bogotá: Tercer Mundo Editores, 2000.

RODRÍGUEZ, L. Una geografía escolar (in) visible. Desarrollo del pensamiento geográfico desde la construcción de conceptos geográficos. Santafé de Bogotá: Ediciones de la Universidad Distrital Francisco José de Caldas, 2007.

SHULMAN, L. Renewing the Pedagogy of Teacher Education: the impact of subject-specific conceptions of teaching. In: VEZ, J. (editor). Las Didácticas Específicas en la Formación del Profesorado I. Santiago de Compostela: Tórculo, 1993, p. 53-69.

SHULMAN, L. Conocimiento y enseñanza: fundamentos de la nueva reforma. Profesorado. Revista de currículum y formación del profesorado, 2005, Vol. 2, № 9. Disponible en internet: http://www. ugr.es/ recfpro/rev92ART1.pdf

SOUTO, X. O interesse da InvestigaÇao na aprendizagem e didática da geografía. In: CASTELLAR, S.; CAVALCANTI, L.; COPETTI, H. Didática da geografía: aportes teóricos e metodológicos. Sao Paulo: Xama Editora, 2012, p. 63-84.

SOUTO, X. Formación del profesorado y didácticas específicas en la educación básica. Revista Electrónica Interuniversitaria de Formación del Profesorado, 2013, Vol. 17, № 1, p. 33-56.

STUART, D. The people's Guide to Spatial Thinking. Washington: National council for Geographic Education, 2013.

YOUNG, M.; LAMBERT D.; ROBERTS, C. \& ROBERTS, M. Knowledge and the future school: curriculum and social justice. British Journal of Educational Studies, 2015, Vol. 63, № 3. Disponible en internet: http://dx.doi.org/10.1080/00071005.2015.1072338

VYGOTSKY, L. Construção do pensamento e da linguagem. São Paulo: Martins Fontes, 2000. 
